\title{
El aprendizaje de la investigación participativa en torno a la memoria histórica comunitaria
}

\author{
Ronald Martínez-Villarreal* \\ * Investigador y docente del Programa de Museos Regionales y Comunitarios, \\ Museo Nacional de Costa Rica; rmartinez@museocostarica.go.cr
}

Recibido: 24 de abril del 2019 Corregido: 24 de julio del 2019 Aceptado: 20 de agosto del 2019

\section{Resumen}

El artículo presenta la propuesta y los resultados de una investigación sobre los procesos de mediación pedagógica en dos experiencias de formación de equipos de gestores comunitarios, quienes investigan la memoria histórica comunitaria con fuentes orales. El estudio fue de corte cualitativo, desde el paradigma interpretativo y del tipo etnográfico. Se indagó el perfil socioeducativo de los participantes, los contenidos, los métodos y las técnicas de aprendizaje, así como los resultados del proceso de mediación pedagógica e investigación participativa. Entre los principales resultados se logra ver en funcionamiento la democracia cultural, la didáctica de la participación y el aprendizaje social, revelándose una serie de aprendizajes socioafectivos, como el trabajo colaborativo, la incidencia comunitaria y la participación efectiva con implicación.

Palabras clave: Educación no formal; aprendizaje a través de la experiencia; memoria colectiva; investigación; participación comunitaria.

\section{Abstract \\ Learning of the participatory research about community historical memory}

The article resumes the proposal and results of a research on the pedagogical mediation processes in two training experiences of community management teams, who investigate the community historical memory with oral sources. The study is qualitative, from the interpretative paradigm and the ethnographic type. Also, the topics investigated are the socio-educational profile of the participants, the learning contents, methods and techniques of learning and the results of the pedagogical mediation process and participatory research. Is possible appreciate how works the cultural democracy, the didactic of participation and social learning, showing a socio-affective learning such as collaborative work, community advocacy and effective participation with involvement.

Key words: Nonformal education; learning through the experience; collective memory; research; community participation.

\section{INTRODUCCIÓN}

En esta experiencia de investigación se analizan los procesos de mediación pedagógica, para la formación investigativa de personas gestoras comunitarias encargadas de recopilar la memoria histórica a través de fuentes orales. 
Las organizaciones comunitarias con las cuales se trabajó fueron el Museo Comunitario Indígena de Boruca, del cantón de Buenos Aires, Puntarenas y el Equipo Investigador de Historia oral de La Cruz, Guanacaste, Costa Rica. Interesó trabajar en los dos contextos, pues en ambos se presenta la necesidad de mejorar los procesos de formación para el tipo de investigaciones participativas.

El análisis se abordó desde los siguientes objetivos específicos:

- Caracterizar el perfil socioeducativo de las personas gestoras comunitarias al participar en proyectos de investigación de la memoria histórica con fuentes orales.

- Identificar la pertinencia y apropiación de los contenidos de aprendizaje de la formación investigativa.

- Describir los métodos y técnicas de aprendizaje, así como las interacciones y vinculaciones de los actores en el proceso formativo.

- Evaluar los resultados de los investigadores comunitarios en la aplicación de los aprendizajes en sus procesos de investigación.

En ambas agrupaciones estudiadas, se desarrollan particulares procesos de investigación participativa, motivados por intereses colectivos por conservar, difundir y gestionar la memoria histórica comunitaria como herramienta de reafirmación de la identidad cultural y de autodeterminación local. Por ende, el proceso formativo que llevan a cabo estas personas es de especial interés, no solo para la misma comunidad, sino porque representa un esfuerzo poco frecuente en nuestro país por apropiarse de herramientas de investigación histórica con fuentes orales, realizado por miembros de la misma comunidad y que expresan su propia voz. Así los resultados del proceso formativo significan una interpretación histórica, por primera vez no vista desde afuera, por un investigador externo, sino desde la misma realidad local, por gestores conectados con su contexto, su memoria e informantes, que más allá de esto son sus vecinos, amigos y familiares.

Durante la investigación, se tomaron en cuenta posturas teóricas como la didáctica de la participación y el aprendizaje social, mediante una metodología cualitativa basada en el paradigma interpretativo y según el tipo de investigación etnográfica.

\section{Sustento teórico}

La experiencia formativa analizada se ubica dentro de la participación como fenómeno comunitario de reivindicación y autodeterminación, lo cual parte de la democracia cultural entendida como uno de los estados avanzados de la gestión cultural donde se busca "catalizar la potencialidad de las comunidades para genera su propia oferta cultural" (Trilla, 2004, p.17).

Bajo este principio, la comunidad cuenta con la capacidad de defender y gestionar su propia producción cultural al mismo nivel de la producción clásica o del Estado, con el aliciente de ser definida y producida desde las formas propias de organización comunitaria. Por ende, se trata de una participación comunitaria ejecutiva (Ventosa, 2016) en donde las personas participantes son responsables de la definición, análisis, toma de decisiones y ejecución de las acciones de investigación histórica con fuentes orales. Esto marca las acciones de mediación pedagógica, en tanto deben ser facilitadoras en apego a la autodeterminación dada por la participación ejecutiva.

En cuanto al ámbito educativo, para este caso se trata de educación no formal. Pero, debe tenerse claro que la educación formal y la no formal conviven y se reconoce ambas como actos intencionales, programados y fundamentados, en donde la función docente debe estar presente siempre con rigurosidad 
(Ortega, 2005). De tal manera, en lo no formal, el espacio que conocemos como aula pasa a ser asumido por el grupo, con sus interacciones y motivaciones propias.

La diferenciación entre los ámbitos citados no debe interpretarse como tajante o arbitraria, pues entre estos conviven relaciones de suplencia, sobre todo cuando la educación no formal asume tareas en las que el sistema formal falla de manera parcial o total. También relaciones de sustitución, como cuando desde lo no formal se atiende un faltante de alfabetización. A su vez, la educación no formal adquiere diversidad de objetivos, finalidades, agentes y formas de ser entregada, por lo cual no reconoce distinciones entre sus destinatarios y termina siendo flexible en cuanto a requisitos y programaciones. Asimismo, puede culminar en titulaciones, al igual que el ámbito formal, y sus contenidos y metodologías "están rociados por la cultura vigente, al mismo tiempo que se adaptan a las particularidades de los sujetos y los contextos donde se realiza la acción pedagógica" (Torres, 2007, p.22).

Aunado a lo anterior, resulta útil también el concepto de educación a lo largo de la vida, postulado por la UNESCO (1997) a partir de la publicación de La Educación encierra un tesoro. Dicho concepto plantea que, "en el espacio y el tiempo de la vida, aprendamos a aprender y conocer, a hacer, a vivir juntos y a ser nosotros mismos" (Ortega, 2005, p.168). Es decir, el desarrollo armonioso y continuo de la persona, requiere una formación que recupere el autoconocimiento del individuo y su entorno, en tanto forma parte de una familia y una ciudadanía con quienes convive y coopera.

\section{La construcción del conocimiento desde la experiencia social}

En otro nivel teórico, la investigación incita al diálogo entre el aprendizaje histórico-social (Vigotsky, citado por Chaves, 2001) y la pedagogía de la liberación propuesta por Paulo Freire (2006).

En el primer caso, el aprendizaje se entiende como una actividad colectiva y de comunicación de la cultura mediante signos, y esto se evidencia en lo que se denomina la zona de desarrollo próximo, concepto que "designa las acciones del individuo que al inicio él puede realizar exitosamente sólo en interrelación con otras personas, en la comunicación con éstas y con su ayuda, pero que luego puede cumplir en forma totalmente autónoma y voluntaria" (Chaves, 2001, p.62).

En cuanto a la pedagogía de la libertad, su promotor Paulo Freire plantea el espacio educativo más allá de una relación maestro-aprendiz, concebida más bien desde lo que él llama círculo de cultura en el cual "ya nadie educa a nadie, así como tampoco nadie se educa a sí mismo, los hombres se educan en comunión, y el mundo es el mediador" (Freire, 2014, p.85). En ese orden, la mediación pedagógica pasa por la creación de un ambiente de grupo, en donde los aportes de todos se integran en un proceso de construcción colectiva de aprendizajes.

Por otra parte, la didáctica de la participación es sustantiva para el presente trabajo, pues con ella se entiende que se trata de una forma de aprendizaje para el desarrollo de la persona a través de la mediación, según el principio de que "la mejor manera de saber lo que es la participación, es participando" (Ventosa, 2016, p.61).

De esta forma, aprender a participar se compone de contenidos procedimentales que potencien la capacidad de los grupos para asumirla, propiciando a la vez actitudes positivas.

Es así, como el fenómeno educativo estudiado se contempla como participativo, no formal y socioformativo. La función docente es asumida por el formador del proyecto y el grupo es el destinatario del proceso en interacción, como se explicará más adelante, con la familia y la comunidad. 
Las interacciones de grupo se hallan a cargo de las personas gestoras comunitarias encargadas de recopilar la memoria histórica a través de fuentes orales, generalmente pertenecientes a colectivos que desde sus organizaciones comunitarias como lo son el Museo Comunitario Indígena de Boruca y el Equipo investigador de Historia oral de La Cruz, asumen labores en donde investigan, escriben, exhiben y difunden la memoria histórica comunitaria, desde bien sea el museo comunitario u otras acciones de interpretación y comunicación de la identidad cultural.

Por último, en este apartado teórico la mediación pedagógica se analiza desde cuatro componentes:

- El perfil socioeducativo de las personas gestoras comunitarias: definido como las habilidades cognitivas, procedimentales y actitudinales, explícitas e implícitas (Baráibar, 2003). Con estas habilidades los participantes asumen su labor de investigadores de la memoria histórica comunitaria con fuentes orales.

- Pertinencia y apropiación de los contenidos de aprendizaje: lo que se refiere a la utilidad de los conocimientos, procedimientos y actitudes desarrolladas durante el proceso formativo para concluir las investigaciones en historia oral, y cómo estos son entendidos y aplicados por los participantes en el desarrollo de sus investigaciones.

- Métodos y técnicas de aprendizaje utilizados en la formación para la investigación: según lo cual, el grupo es el destinatario de la formación, entendido como colectivo que en sus cohesiones, intereses y metas es también constructor del proceso formativo y realizador de la investigación para la cual se forma (Izquierdo \& Izquierdo, 2009).

- Los resultados de los investigadores comunitarios en la aplicación aprendizajes: se refiere a las informaciones que los participantes logran recopilar y disponer, mediante la realización de entrevistas, las cuales permiten responder y explicar el guion de entrevista, así como atender a los objetivos del proceso formativo de investigación histórica con fuentes orales.

\section{METODOLOGÍA}

La investigación se efectuó a partir del paradigma interpretativo, también conocido como naturalista o participativo. Se buscó, desde el enfoque cualitativo, analizar cómo los gestores-investigadores comunitarios ofrecen una percepción, reacción y resultados sobre su proceso formativo y la aplicación de los aprendizajes en la investigación de la memoria comunitaria con fuentes orales. Al sistematizar los hallazgos, se conoció y analizó el impacto del proceso formativo en cuanto a la construcción participativa de un proceso investigativo de impacto comunitario.

Así, se indagaron y recolectaron datos concernientes a los conocimientos, las perspectivas, los resultados y los puntos de vista de los participantes y se sistematizaron los planos subjetivos en tendencias y descriptores. Para ello se utilizaron categorías de análisis desprendidas de los objetivos específicos, desde las cuales se detallan "situaciones, eventos, personas, interacciones, conductas observadas y sus manifestaciones" (Hernández, Fernández y Baptista, 2014, p.9).

Se optó por un tipo de investigación etnográfica, al explorarse el fenómeno educativo de la formación mediante la investigación con fuentes orales desde el significado y función de las acciones de los sujetos (Gurdián-Fernández, 2007). Verbalizadas las acciones por medio de descripciones y explicaciones, así como indagadas en técnicas colectivas como el grupo de discusión e individuales como la entrevista enfocada y la observación participante.

En cuanto a los participantes del estudio se trata, para el caso de Boruca, de miembros de la Asociación de Artesanos La Flor de Boruca, los cuales en acuerdo con la Asociación de Desarrollo Indígena son 
los encargados de administrar de forma participativa e integradora el Museo Comunitario de Boruca. Participaron ocho miembros del museo comunitario, seis mujeres y dos varones, quienes han llevado los procesos formativos y han desarrollado investigaciones con fuentes orales para la creación del guion de exhibición del museo comunitario y la construcción de una memoria histórica comunitaria, ambos productos facilitados por el Programa de Museos Regionales y Comunitarios del Museo Nacional de Costa Rica.

En cuanto a La Cruz, los sujetos de información fueron seis miembros del equipo investigador de historia oral de La Cruz, cuatro mujeres y seis hombres, cuya conformación ha sido impulsada en coordinación entre el área de gestión cultural municipal y el centro universitario de la UNED. Con el grupo se ha llevado un proceso formativo y de trabajo de campo para recopilar la memoria histórica comunitaria con fuentes orales, en el marco del Proyecto Historial Oral Participativa en La Cruz de la UNED.

La cantidad de personas permitió trabajar con la totalidad de los sujetos, contando con muy buena respuesta de asistencia a los trabajos relativos a la memoria histórica de la comunidad.

Para las técnicas de recolección de la información, se utilizaron los grupos de discusión, las entrevistas enfocadas y la observación participante. Los grupos de discusión se diseñaron proponiendo la creación de un ambiente dinámico y lúdico, pero a la vez riguroso en cuanto a la captura de datos cualitativos que lograsen describir y explicar las categorías de análisis. Las entrevistas fueron igualmente diseñadas a partir de las categorías de análisis y generaron un cuerpo de datos cualitativos, paralelo a los resultados de los grupos de discusión, esto para su comparación y contrastación. Por último, en la observación participante se utilizó una guía de observación para acompañar a uno de los equipos de investigación durante una entrevista de recopilación de memoria histórica, con el fin de capturar datos cualitativos atinentes.

Para el análisis e interpretación de los datos, se construyó una matriz que permitió la organización de las fuentes recolectadas, para luego comparar y cruzar los hallazgos de cada técnica y fuentes de información con la respectiva categoría de análisis (Figura 1). Esta parte del procedimiento se facilitó con la codificación de las categorías y su análisis asistido por el software de análisis cualitativo de datos AtlasTi®. De esta manera se triangularon los resultados obtenidos, junto con las acotaciones teóricas correspondientes.

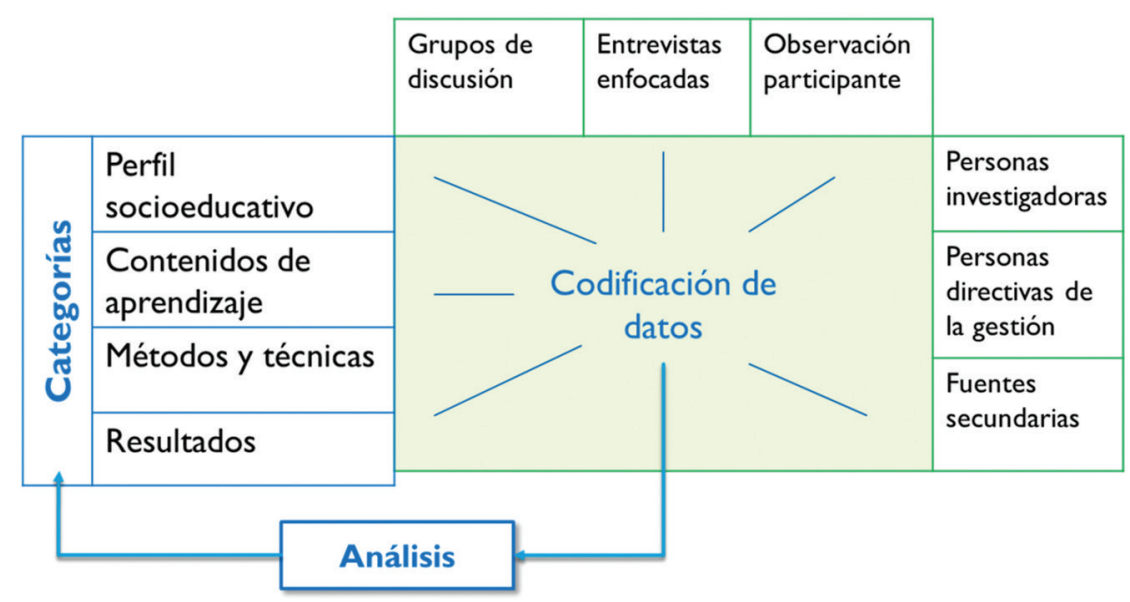

Figura 1. Estrategia para el análisis e interpretación de datos. Fuente: Martínez (2018). 


\section{RESULTADOS}

\section{Perfil socioeducativo de las personas gestoras comunitarias al participar en proyectos de investigación}

El componente se entiende como las habilidades cognitivas, procedimentales y actitudinales, explícitas e implícitas mediante las cuales los participantes asumen su labor de investigadores de la memoria histórica comunitaria con fuentes orales.

En ese sentido, las personas gestoras comunitarias se caracterizan por el compromiso e interés por investigar su memoria histórica comunitaria, y por ello asumen la realización de un proyecto que busca la atención de una necesidad colectiva: fortalecer la identidad cultural y las raíces históricas locales.

El compromiso demuestra una experiencia de democracia cultural, pues aprender a investigar les permite a las personas gestoras comunitarias participar activamente, al asumir por sí mismas un proyecto de impacto local que les permite decidir y aprender-haciendo, lo anterior apoyado por una mediación pedagógica más allá de la sola apropiación de contenidos.

En este proceso de investigación participativa, el discurso resultante sobre la memoria histórica comunitaria es una construcción colectiva, producto de las relaciones de convivencia entre los investigadores y su propia comunidad. A su vez representa un aprendizaje de la convivencia, donde se valora a las personas y las relaciones que les rodean, lo cual fortalece los lazos comunitarios de identidad.

Los participantes del proceso formativo desarrollaron una particular motivación al sentirse protagonistas del desarrollo local, revelando lo que Paulo Freire (2014) llamó "la conciencia intencionada del mundo", la cual permite al individuo trascender de sí mismo. Aprender de así presenta una inspiración y recompensa diferente, una utilidad para el crecimiento de la persona, de la comunidad y en general en la "humanización del mundo" (Freire, 2016).

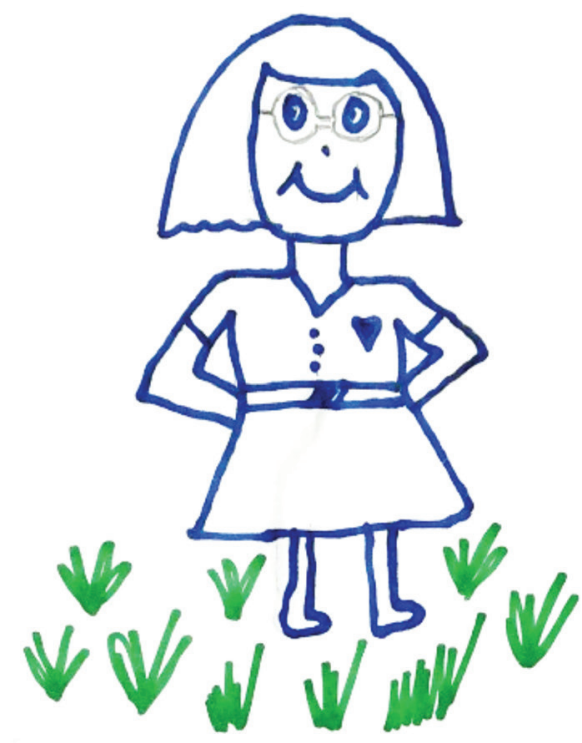

Figura 2. Representación de la persona investigadora. Fuente: Martínez (2018). 
Estas consideraciones sobre el perfil socioeducativo quedan expresadas tanto en la figura anterior como en el siguiente extracto expresado por una de las personas investigadoras.

"[...] percibo una persona pues si con la carita ya pasada en años por las fracciones de la sonrisa que tiene verdad, se le ve una sonrisita o sea es sonriente, pero ya los años ya han caído en ella, ama lo que va a hacer porque el corazón lo lleva ahí[...]hasta que le "flota"[...] risas...y si es una persona como ya pasada en años, la veo así ya, ya muy muy centrada en lo que va a hacer y va muy feliz en el campo[...] risas[...]" (Grupo de discusión, La Cruz, 2018)

También surgió dentro de las personas gestoras el fenómeno de la implicación, según lo que Ventosa (2016) define como la posibilidad de aprender a participar-participando y cómo los resultados de esta acción permiten a la persona involucrada sentirse útil por realizar una acción valiosa para la comunidad.

Por ende, la persona participante cree en la convivencia, el valor de aportar su tiempo y sus esfuerzos a la comunidad, aprendiendo y actuando para el cambio. Las personas gestoras además reconocieron como habilidades indispensables, la responsabilidad, la sabiduría, la paciencia y el empoderamiento para poder involucrarse en un proceso formativo. Esto evidencia la búsqueda de una persona nueva, un individuo capaz de impactar positivamente en su comunidad, tal y como se aprecia en las siguientes evidencias.

"[...] echamos mano también de la confianza que tenemos que generar a esa persona para que nos hable de su vida porque en resumen nos va a hablar bastante de su vida personal" (Grupo de discusión, La Cruz, 2018).

“Es increíble también el ánimo del momento de uno, yo en ningún momento tuve miedo de enfrentarme a ella, de llegar donde ella y tener la confianza de poder este entablar una conversación" (Entrevista, Boruca, 2018).

"En todo momento la conversación fluye. Se logra sacar provecho de que la persona entrevistada se siente alagada por la visita y el lugar que se le da" (Observación, La Cruz, 2018).

En el proceso, se aprende a aplicar entrevistas, pero más allá de lo cognitivo, la entrevista es vivida como un encuentro, una convivencia. Por ende, se requiere de personas investigadoras con la voluntad y la convicción de mejorar su contexto.

Ante este panorama, el proceso formativo tiene la tarea de canalizar estos perfiles de las personas en la aplicación de procesos exitosos de investigación histórica y participativa con fuentes orales.

\section{Contenidos de aprendizaje para la formación investigativa de las personas gestoras comunitarias}

Aquellos contenidos que se pueden denominar como de aplicación efectiva fueron dos: 1) presentación e inicio de la entrevista; y 2) generación de un ambiente que facilite la conversación. Las personas gestoras manifestaron confianza en su dominio y esto se logró en una zona de desarrollo próximo colaborativa, la cual propicia el tránsito desde las acciones conocidas y de confianza hasta alcanzar conocimientos nuevos, proceso apoyado por acompañamiento conjunto, propio del aprendizaje social.

A su vez, cuando esos contenidos muestran alguna dificultad, más que desánimo se convierten en retos a la zona de confort. De entrada, los participantes pueden no saber cómo hacerlo, pero son conscientes 
de que es relevante intentar su dominio para desarrollarse como personas y ayudar a la comunidad, cultivando nuevas competencias.

Por otra parte y como se ve en el siguiente extracto de entrevista, las dificultades y frustraciones que implica el manejo de equipos tecnológicos para las grabaciones y el almacenamiento de datos, surgieron al no contar con una propuesta didáctica que capacite a las personas gestoras en el adecuado uso de estas herramientas.

"A mí lo que no me gustó es no tener conocimiento de un manejo tecnológico, ya sea computadora y esas tonteras, y no pude guardar mis entrevistas, y al final todo lo perdí, todo se fue y no puedo tampoco volverlos a recuperar, entonces es eso para mí una lástima, porque diay, en el momento en que fuimos a hacer las entrevistas fue algo muy bonito para nosotros y ya queda solo en la memoria de nosotros nada más que fuimos porque no tengo donde a donde, porque la audio y todo se fue." (Entrevista, Boruca, 2018)

Si bien se trata de un proceso formativo de orientación histórica, cultural y social, no se puede obviar la formación de destrezas tecnológicas. Los participantes identificaron esta limitante y reconocieron a la vez como posible solución pedir ayuda a otros compañeros con más habilidades en el área.

Los contenidos que se identifican como verdaderamente apropiados o de menor dificultad son aquellos que se emplean para el logro de soluciones durante la investigación (cómo definir temas de investigación, presentación de la entrevista, historia oral, crear ambiente de conversación, guía de entrevista). De esta manera, los elementos teóricos demuestran utilidad cuando se les necesita también dentro del proceso de investigación.

El proceso de apropiación se evidencia cuando los participantes toman conciencia de lo que son capaces gracias al proceso formativo y los resultados del proyecto en que participan. El fenómeno citado se explica mediante el siguiente esquema.

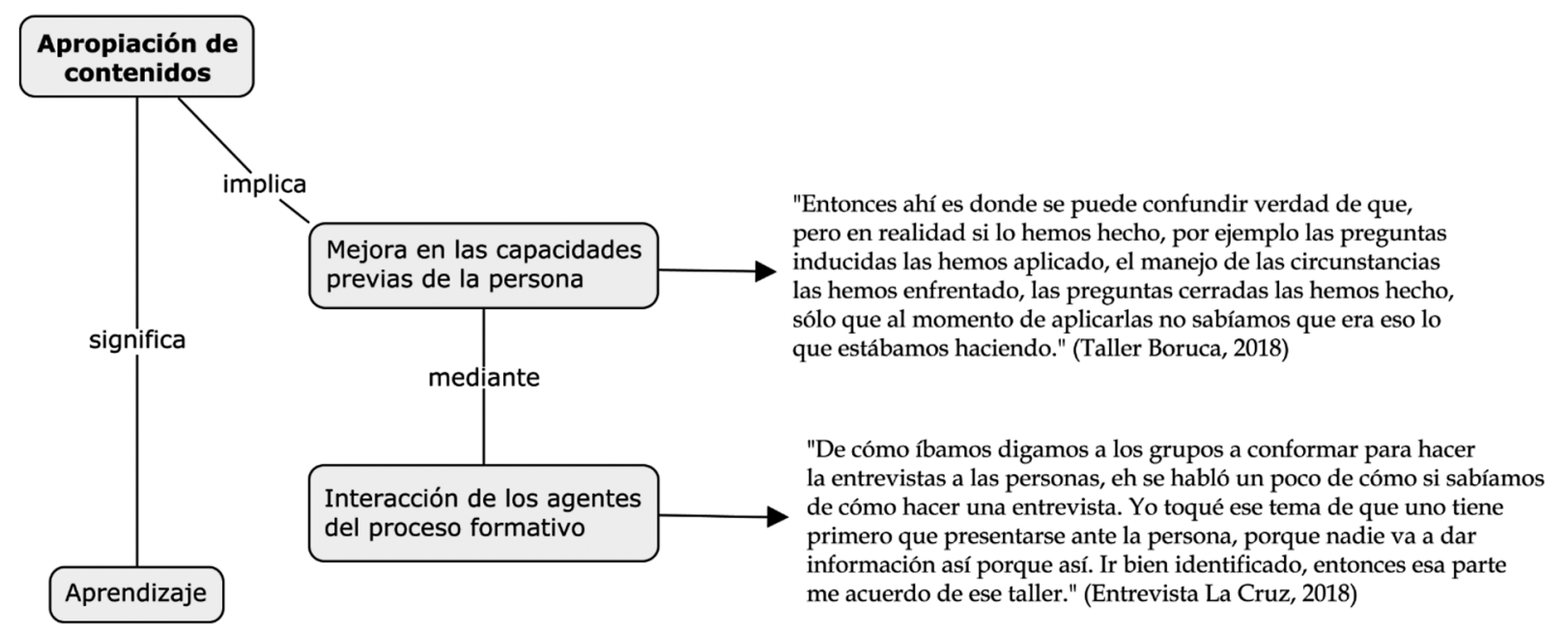

Figura 3. Construcción del proceso de apropiación de contenidos de aprendizaje Fuente: Martínez (2018). 
Por último, para las personas gestoras la experiencia de gestionar un proyecto de investigación histórica resultó también ser parte del proceso formativo. Así, el aprendizaje pretende ser transversal y constante, pues se aprende según se avanza en el proyecto de forma implícita e inevitable.

\section{Métodos y técnicas de aprendizaje utilizados en la formación investigativa}

El círculo de cultura sobresale como método a través del cual se desarrolla esta formación. Según su creador, Pablo Freire (2014) el método desarrolla un espacio humanizante y liberador de aprendizaje mediante la reflexión, el diálogo y la colaboración. Una reflexión que conduce a la práctica, el diálogo como una oportunidad de encuentro para la reflexión y la acción hacia el mundo que debe de ser transformado y humanizado y la colaboración que construye responsabilidades asumidas con motivación y con ilusión de los sujetos que quieren descubrir la capacidad de transformar su realidad a través de su trabajo creador.

De esta manera, hay una serie de logros que en el proceso investigativo quedan en evidencia, con ello los participantes descubren nuevas capacidades que a la vez los animan a seguir adelante. En concreto, reconocen la capacidad de hacer una entrevista, en otras palabras, de aplicar el arte de preguntar y en el camino aprender a convivir con las personas adultas mayores de la comunidad a quienes entrevistaron.

El círculo de cultura surge entones, como un método de aprendizaje colectivo a través del diálogo, que permite construir nuevos y valiosos aportes dentro del entorno social inmediato. Esto se evidencia en lo mencionado por las personas gestoras:

"[...] ahí vamos a hacer que las personas despierten su memoria y sus pensamientos abran su cabecita verdad, luego de que tenemos juego de inicio, nos agarramos y de las manos, nos miramos todos en un círculo que es la manera de relacionarnos como seres humanos a través de las manos, sentir el calor, el afecto, compartir porque para hacer una relación interpersonal, de ahí nos presentamos, damos nuestros nombres y hacemos la relación que se llama motivación, hacemos una motivación al grupo, luego de eso explicamos la dinámica del día, qué vamos a hacer, cómo lo vamos a hacer, en cuanto tiempo lo vamos a hacer, qué es lo que tenemos que aprender." (Taller Boruca, 2018).

"[...] sentados en un círculo, compartiendo, cada quién daba su opinión de cómo podíamos[...] sugerencias. A quien se podía entrevistar, a quien no." (Entrevista La Cruz, 2018)

El otro método que se identificó con claridad fue el de Formación en Base en Proyectos (FBP), expuesto por Víctor Ventosa (2016), el cual se sustenta en aprender-haciendo desde lo colectivo, dando un verdadero significado y fin al concepto de participación efectiva, y cuya aplicación lleva al desarrollo de acciones y objetivos de mejoramiento colectivo: en este caso, la construcción participativa de una memoria histórica comunitaria mediante fuentes orales.

De esta forma el FBP, junto al proceso formativo, posibilita el desarrollo de proyectos que apuntan, en último término, a incidir en el contexto social. El proyecto es un proceso con una meta clara, pero en su camino y en sus resultados se genera también el proceso formativo. Si se buscara algún tipo de evaluación sumativa para calificar la formación que esta estrategia propicia, sería entonces mediante el grado de cumplimiento de la investigación de la memoria.

Este tipo de proyectos propician el aprendizaje de contenidos para investigar, pero a la vez se organizan como un trabajo colectivo, lo cual permite aprender cómo organizarse y cómo gestionar el proyecto en sí. 


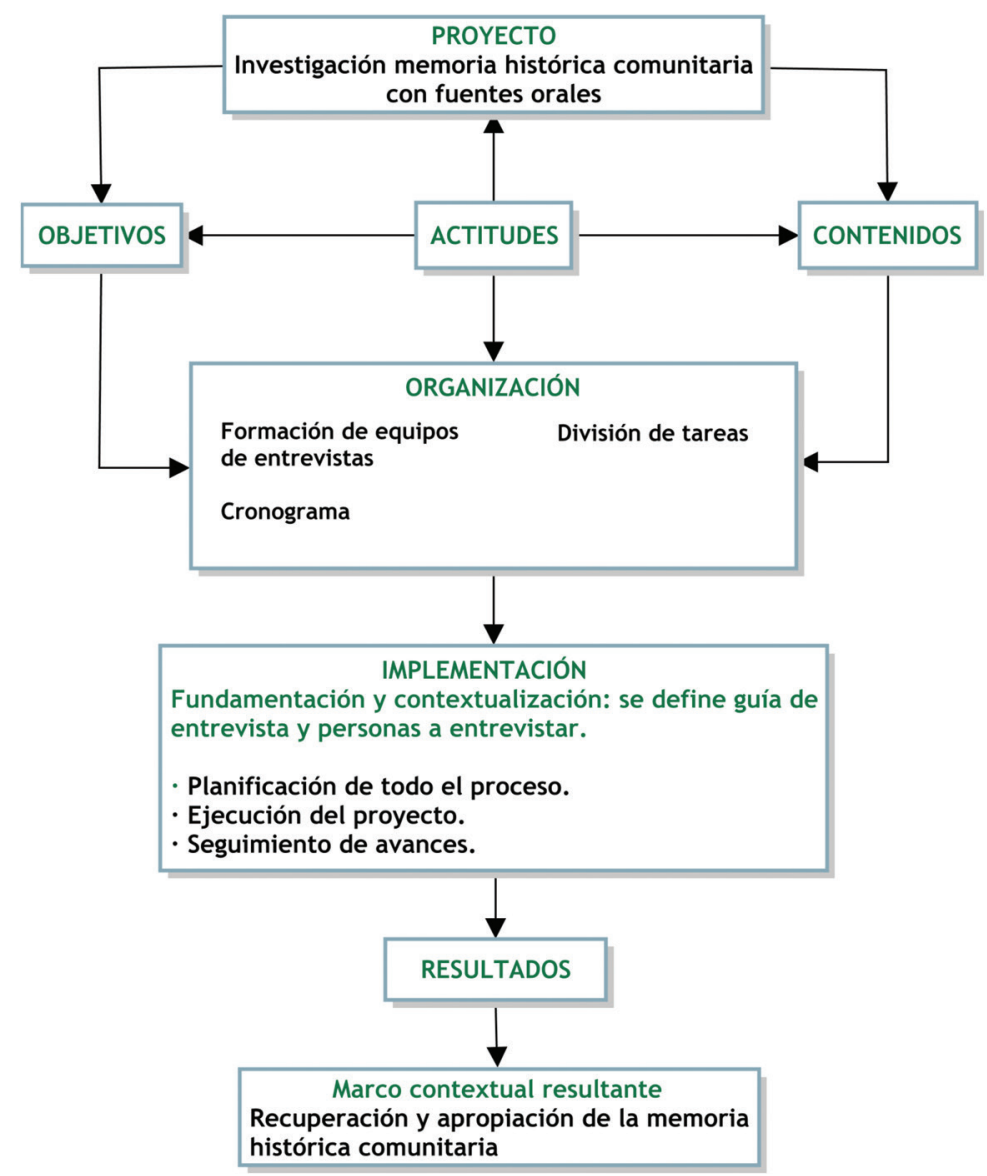

Figura 4. Modelo de Formación en Base en Proyectos aplicado al análisis del proceso formativo. Fuente: elaboración propia basado en Ventosa (2004).

En cuanto a las técnicas de aprendizaje, el grupo de personas gestoras, en sus relaciones y funcionamiento, sobresale como el medio a través del cual sucede el aprendizaje, el cual es exitoso cuando se visualizan sus logros. En el trabajo grupal se presenta el estudio de casos que forman parte de la misma implementación del proyecto de investigación, por lo que constituyen aprendizajes llevados a la acción a través de vivencias propias del contexto de cada comunidad.

Asimismo, en estos espacios de aprendizaje grupal, se suscitan relaciones entre la persona formadora y los participantes. Según los resultados arrojados, el formador asume un riesgo cuando toma posturas laizes-faire y paternalistas (Casellas, 2003, p. 293), pues el grupo puede caer en la dependencia y se debilita la participación y el liderazgo. Además, debe también cuidarse cuando de forma muy reiterada responde meramente a la situación o momento grupal (estilo situacional), desviándose el proceso de los objetivos formativos.

Es así como, en el grupo se aprende a equilibrar emociones y discrepancias, para así mantener el enfoque en la tarea. El mantenimiento de este equilibrio significa un contenido de aprendizaje socioafectivo, el cual es clave para conservar la concentración en el logro del proyecto. Sin embargo, el estudio no arrojó evidencias de cómo la persona formadora propone la construcción del aprendizaje socioafectivo.

Finalmente, se observó el desarrollo de un aprendizaje social que pretende ser efectivo y vivencial en la búsqueda del logro del objetivo del proyecto comunitario. 
Es, entonces, un aprendizaje a favor del desarrollo social, dirigido a responder a una necesidad llamada memoria comunitaria, y en cuya atención los participantes se vuelven mejores personas al participar activamente de una comunidad. El aprendizaje sucede en tres niveles de experiencia social, como son el familiar, el comunitario y el intergeneracional, aprovechando los afectos, la confianza, la colaboración y el respeto que se desarrollan en el contexto. Dicha construcción de aprendizaje social detectada en esta investigación, se sintetiza en la siguiente figura.

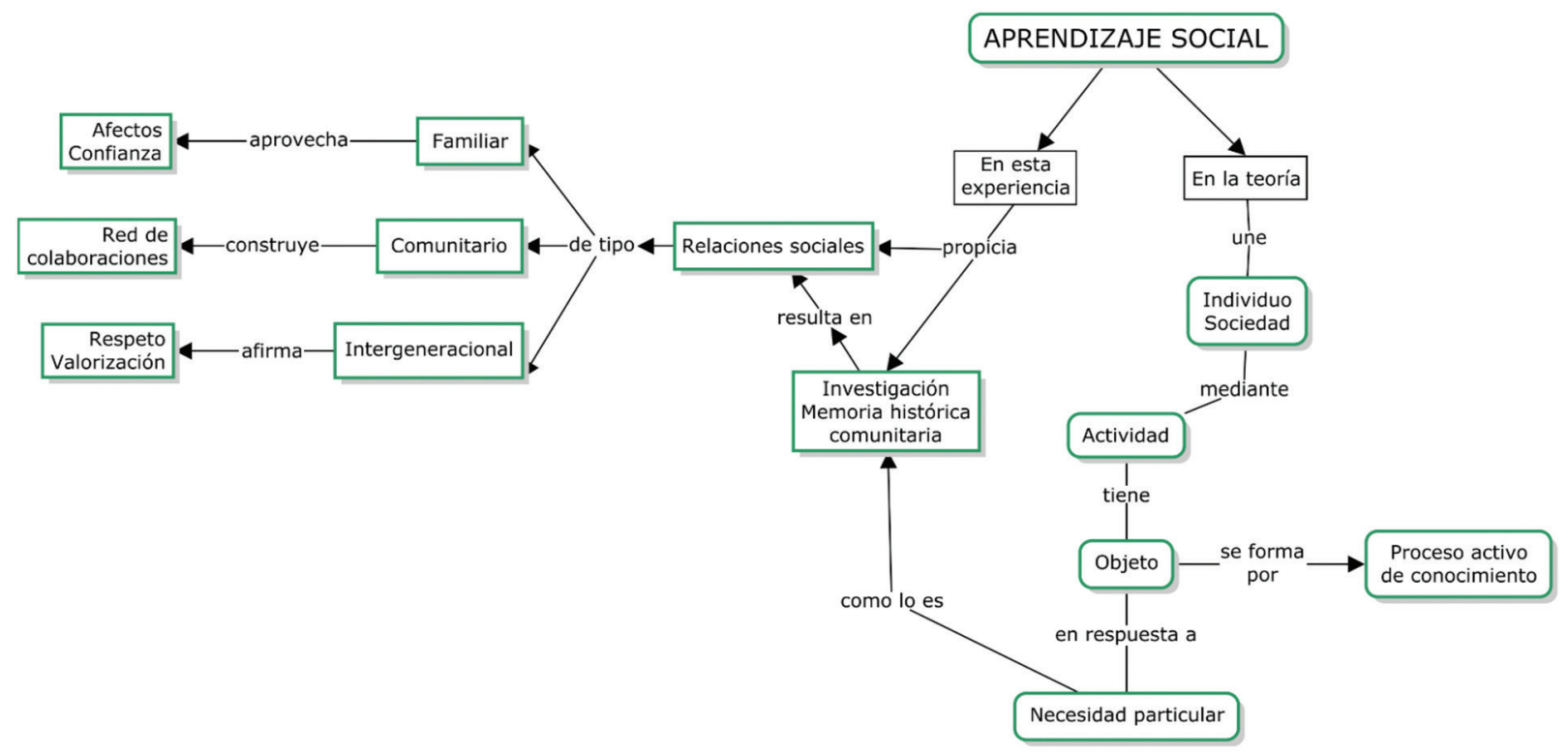

Figura 5. Construcción del aprendizaje social en la investigación de la memoria histórica comunitaria.

Fuente: Elaboración propia con base en Garzón, 2007 y resultados del análisis.

Por último, llama la atención que, según los datos analizados, en el proceso formativo no se evidencia el uso de algún material de apoyo para los diferentes talleres como separatas o textos, con lo cual no es posible saber si este tipo de recursos incide de alguna manera en la mediación educativa analizada.

\section{Resultados obtenidos por los investigadores comunitarios tras la aplicación de los aprendizajes}

El primero de los resultados reconocidos en el análisis corresponde al plano socioafectivo, pues las personas gestoras comunitarias participantes desarrollan un significativo compromiso comunitario y dedicación por el bienestar común, lo que puede señalarse como un tipo de efecto humanizante de la educación.

Al analizar los logros cognitivos, resulta necesario profundizar más en contenidos como: 1) la buena definición de los temas por investigar y 2) las disconformidades expresadas con la cantidad y calidad de información recopilada. Esto aunado a las deficiencias detectadas en el manejo del equipo de grabación, las cuales no se llevan al plano de la convivencia y el compromiso comunitario para ser resueltas (por ejemplo, que otros miembros de la comunidad con destrezas en el manejo de herramientas técnicas sean integrados al proceso investigativo). 
Por otra parte, el uso de la guía de preguntas puede ser el medio a través del cual se puedan mediar estas deficiencias, por tratarse de una herramienta proveniente del plano cognitivo, la cual permite interacciones y logros socioafectivos que, como ya se mencionó, suceden en el proceso de la entrevista.

\section{CONCLUSIONES Y RECOMENDACIONES}

De acuerdo con los objetivos de investigación, se lograron las siguientes conclusiones.

Primeramente, en cuanto al perfil socioeducativo de las personas gestoras comunitarias que participan de proyectos de investigación de la memoria histórica, se evidenció como el investigador no es solo un indagador, sino también un sujeto implicado para el mejoramiento comunitario, capacitado en cuanto a respeto, responsabilidad, sabiduría y paciencia y fiel al servicio del fortalecimiento del sentido de identidad local. Además de conocedor y parte de las interacciones sociales que posibilitan mantener viva la memoria histórica comunitaria. El perfil del investigador se construye entonces en un aprender a investigar en la convivencia.

Por su parte, en relación con los contenidos de aprendizaje de la formación investigativa, los saberes identificados con mejor dominio fueron la presentación e inicio de la entrevista y la generación de un ambiente que facilite la conversación. Favorecida su apropiación por una zona de desarrollo próximo que parte de las habilidades de comunicación entre vecinos y se apoya en la colaboración mutua de participantes, la motivación y el aprendizaje social.

Por el contrario, los contenidos más difíciles se relacionan con la grabación y almacenamiento de datos recopilados en las entrevistas, producto de falta de habilidades tecnológicas o indisposición de enfrentarse a estas, pero siendo conscientes de que deberían apoyarse en compañeros con más experiencia al respecto.

Ante todo, el tomar conciencia sobre lo aprendido reveló los logros en la apropiación de contenidos y por ende de aprendizajes. Los participantes piensan como habilidades la entrevista y la creación de un ambiente de conversación fueron mejoradas, siendo esto posible a través de la misma interacción y solidaridad del grupo.

En lo relativo a los métodos y técnicas de aprendizaje utilizados en la formación investigativa de la memoria histórica a través de fuentes orales, el método privilegiado fue el círculo de cultura, desarrollado a través de relaciones grupales de diálogo, reflexión y colaboración, en donde en conjunto se construyen convicciones de estar transformando y humanizando el mundo, entregando a la comunidad su memoria histórica para su conservación, autodeterminación y expresión de la identidad cultural.

Lo anterior, funciona de la mano con el método de Formación en Base a Proyectos (FBP), el cual imprime un sentido tangible y significativo al aprendizaje y a la investigación, pues a lo largo del proyecto se van identificando avances, logros, cambios y aprendizajes, junto a resultados de investigación conforme se avanza con las entrevistas y la sistematización.

Todo lo anterior en un marco de aprendizaje social, en donde los participantes aprenden investigando su memoria en una mezcla de interacciones sociales de tipo familiar, comunal e intergeneracional.

Por último, en cuanto a los resultados de los investigadores comunitarios en la aplicación de los aprendizajes en los procesos de investigación con fuentes orales, el resultado mejor evidenciado es que los participantes logran una interpretación propia de su historia común, construida desde la convivencia comunitaria en la que se convierte el proceso de aprendizaje e investigación. El esfuerzo de investigación cobra entonces un sentido de utilidad diferente y genera en los involucrados una satisfacción de aporte y entrega al bienestar común. 
Este convencimiento debe ser canalizado desde el principio de los procesos como motivación para involucrarse a participar y así construir de manera colectiva un acuerdo de trabajo, con su ruta de aprendizaje y acción, es decir: cómo se va a trabajar, cuál va a ser el método y la dinámica de grupo.

Eventualmente, la propuesta es que esta investigación funcione a modo de un diagnóstico del proceso formativo para la investigación de la memoria histórica comunitaria, el cual sirva como insumo para proponer y validar una propuesta didáctica de investigación histórica participativa; de tal forma que estos resultados se apliquen al mejoramiento de la propuesta formativa que actualmente se está llevando a cabo.

De esta manera, el presente diagnóstico propone insumos para avanzar a otras etapas de propuesta pedagógica y consolidar un ámbito que pueda ser útil a los gestores comunitarios y docentes, tanto formales como no formales, de instituciones y organizaciones que quieran promover este tipo de experiencias docentes.

El estudio se plantea como un aporte para que a nivel comunitario se consolide la participación en la investigación histórica y la gestión cultural, promoviendo así la autodeterminación de las personas y los pueblos. De esta manera, la didáctica de la participación podrá ser llevada a un nuevo espacio de docencia como lo es la formación para la investigación histórica, asumido hasta hora en mayor medida desde ámbitos académicos.

\section{REFERENCIAS}

Baráibar, J. (2003). Análisis de necesidades formativas. En Lamata Cotanda, R., \& Domínguez Aranda, R. (Ed.). La construcción de procesos formativos en educación no formal. Madrid, Narcea Ediciones.

Chaves, A. (2001). Implicaciones educativas de la teoría sociocultural de Vigotsky. Revista Educación, septiembre, 59-65.

Freire, P. (2006). Pedagogía de la autonomía: saberes necesarios para la práctica educativa. México: Siglo XXI Editores.

Freire, P. (2014). Pedagogía del oprimido. Buenos Aires: Siglo XXI Editores.

Gurdián-Fernández, A. (2007). El Paradigma Cualitativo en la Investigación Socio-Educativa. San José: Coordinación Educativa y Cultural Centroamericana (CECC) - Agencia Española de Cooperación Internacional (AECI).

Hernández, S., Fernández Collado, C., y Baptista, L. (2014). Metodología de la Investigación. México: Editorial McGraw-Hill.

Izquierdo, M. \& Izquierdo, A. (2010). Enseñar a investigar: una propuesta didáctica colaborativa desde la investigación-acción. Documentación de las ciencias de la información, 33, p. 107 - 123.

Martínez, R. (2018). Los procesos de mediación pedagógica para la formación investigativa de las personas gestoras comunitarias encargadas de recopilar la memoria histórica a través de fuentes orales en el Museo Comunitario Indígena de Boruca del cantón de Buenos Aires, Puntarenas y en el Equipo investigador de Historia oral de La Cruz, Guanacaste, durante el primer semestre 2018. Trabajo Final de Graduación, presentado para optar por el grado de Licenciatura en Docencia. Universidad Estatal a Distancia.

Ortega, J. (2005). La educación a lo largo de la vida: la Educación social, la educación escolar. Revista de educación n³38. Educación no formal, (338), 167-175.

Torres, C. (2007). Conceptualización y caracterización de la educación no formal. En Torres, C \& Pareja, J. (Comps.) La educación no formal y diferenciada. Madrid: Editorial CSS. 
Trilla, J. (2004). Concepto, discurso y universo de la Animación Sociocultural. En Trilla, J. (Ed.) Animación Sociocultural. Teorías, programas y ámbitos. Barcelona: Ariel.

UNESCO (1997). La Educación encierra un tesoro. Informe de la UNESCO de la Comisión Internacional sobre la Educación para el siglo XXI, presidida por Jacques Delors. México: UNESCO.

Ventosa, V. (2004). Métodos activos y técnicas de participación para educadores y formadores. Madrid: Editorial CCS.

Ventosa, V. (2016). Didáctica de la participación: teoría, metodología y práctica. Madrid: Narcea ediciones. 